

\title{
Modeling and Analysis of the Wastewater Treatment Plant Operating Costs Based on Oxidation Ditch Process $\dagger$
}

\author{
Liai $\mathrm{CHEN}^{1}$, HongXun Hou${ }^{2}$ and JiaQuan WANG ${ }^{1, *}$
}

${ }^{1}$ School of Management, Hefei University of Technology, Hefei 230009, Anhui Province, P.R. China

${ }^{2}$ Hefei Wastewater Treatment Technology Research Center, Hefei 230088, P.R. China

*Corresponding author: E-mail: jiaquan.wang@163.com

AJC-13339

\begin{abstract}
In order to further research the influence factors of total costs and reduce power consumption of wastewater treatment plants, based on the long-term operation data of one wastewater treatment plant, the wastewater treatment costs structure and the operation costs influence factors were analyzed. Combining with the mechanism of biological wastewater treatment, the mathematical model that representing wastewater treatment costs as well as sludge disposal costs was deduced and the statistics test verified its usability. The results demonstrated that: (1) the key factors which effected plant total costs were power consumption and sludge disposal costs; (2) Unit power consumption was approximately proportional to sludge retention time within a certain range; (3) Within a flow range of design, the marginal operating costs would reduce as the increasing of sewage quality in sewage treatment plant; (4) The mathematical model of the relationship between sewage treatment costs and power consumption and sludge disposal costs was established. It indicates that we should adopt a large amount of aeration high sludge retention time, thus the sludge will stabilize in the aeration tank when the electricity was in valley. On the contrary, lower aeration rate and lower sludge retention time will be adopted to achieve the lowest total costs of processing when the electricity was in peak.
\end{abstract}

Key Words: Wastewater treatment plant, Operating costs, Mathematical model, Oxidation ditch, Sludge retention time.

\section{INTRODUCTION}

In the $21^{\text {st }}$ century, under the influence of the growing global water crisis, water pollution problem already become a limiting factor in the global economic sustainable development ${ }^{1}$ and it will become the most prominent problem of the economic development. Realizing wastewater reutilization can be obviously beneficial to environmental, economic and social benefits, which is also the effective measure to protect water resources and value-added and it can greatly ease water shortage $^{2}$. Wastewater treatment plant is one of the key links enabling regeneration and recycling of wastewater.

Among the wastewater treatment plants, oxidation ditch process rank first which take up $28.5 \%$ in number and $20.5 \%$ in treatment ability ${ }^{1}$. It is efficient for wastewater treatment ${ }^{3,4}$, but it has high energy consumption ${ }^{5,6}$. Recently, operating costs of the wastewater treatment plant are much concerned in china due to the large treatment wastewater quantity ${ }^{7,8}$ and relating to costs which is given more and more attentions in recent years $^{9-11}$. But no one gives a mathematical model of wastewater treatment plant to reduce it.
The paper took one of wastewater treatment plants in Hefei city for an example, a mathematical model on which could reduce costs effectively was first put forward, that can be both a response to the government target "saving energy and reducing consumption".

The rest of this paper was as follows: after the introduction of the wastewater treatment plant, the process of modeling of the wastewater treatment costs was outlined with statistical test of the result. Then the relationship between total costs of wastewater treatment and power costs and costs of sludge disposal was deduced with the detailed analysis. Finally, before and after transformation, energy consumption was described.

Costs composition of the wastewater treatment plant: In the wastewater treatment plant, anaerobic-anoxia-oxidation ditch process was adopted with a design scale $100,000 \mathrm{~m}^{3} \cdot \mathrm{d}^{-1}$. The theoretical hydraulic retention time for anaerobic tank, anoxia tank and aeration tank was $1.5 \mathrm{~h}, 0.8 \mathrm{~h}$ and $9.6 \mathrm{~h}$, respectively. The accurately hydraulic retention time was 1.3 h, $2.4 \mathrm{~h}$ and $15.7 \mathrm{~h}$ respectively. Tank depth was $4.5 \mathrm{~m}, 6$ inverted umbrella aerator machines with input power of 150 $\mathrm{kW}$ were used. 
The daily processing costs of wastewater treatment plant included the following several aspects ${ }^{12}$ : (1) Wastewater disposal power costs; (2) Wastewater disposal reagents; (3) Sludge disposal costs; (4) Tap water costs ; (5) Equipment maintenance costs; (6) Employee costs, etc. If only consider the above 6 items costs, the components part of wastewater treatment plant daily processing costs are shown in Fig. 1.

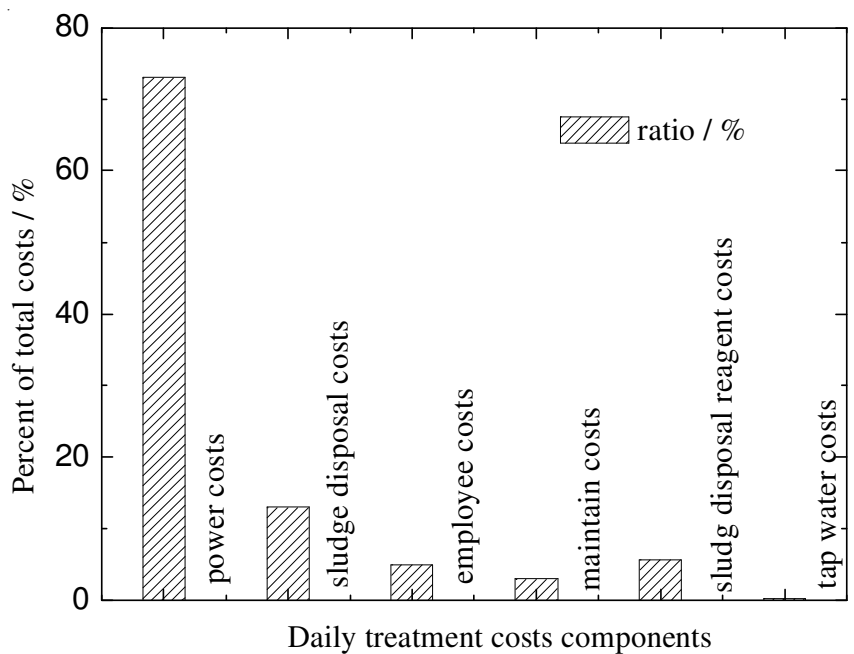

Fig. 1. Daily treatment costs components of the wastewater treatment plant

As presented in Fig. 1, power costs and sludge disposal costs amount to $86 \%$ of daily processing costs. Employee costs and equipment maintenance costs only have slightest impact on daily processing costs and tap water costs which can be neglected relative to the power costs and water sludge disposal costs. These results were consistent with the literature reported ${ }^{13,14}$. For the majority of wastewater treatment plants, reagent costs can be contained in sludge disposal costs because they are mainly used for wastewater disposal. Based on the above analysis, we could reach a conclusion that the daily processing costs are mainly composed of 2 parts: variable costs that contain wastewater disposal power costs (A) and wastewater disposal costs (B), fixed costs (C) that contain salary costs, reagent costs, tap water costs, etc.

Modeling of wastewater treatment costs: The relationship between the total costs of wastewater treatment per unit and power costs per unit and costs of sludge disposal produced by unit wastewater was given by eqn. (1):

$$
\mathrm{T}=\mathrm{A}+\mathrm{B}+\mathrm{C}=\mathrm{ax}_{1}+\mathrm{bx}_{2}+\mathrm{c}
$$

where the variables respectively stand for: $\mathrm{T}$, water total costs per cubic metre $\left(\mathrm{Y} \cdot \mathrm{m}^{-3}\right)$; A, wastewater disposal power costs; $\mathrm{B}$, wastewater disposal costs; $\mathrm{C}$, other fixed costs. a, power price $\left(\mathrm{Y} \cdot \mathrm{k}^{-1} \cdot \mathrm{W}^{-1} \cdot \mathrm{h}^{-1}\right) ; \mathrm{b}$, quantity of excess sludge produced by unit wastewater $\left(\mathrm{t} \cdot \mathrm{m}^{-3}\right)$; $\mathrm{c}$, the same with $\mathrm{C}$; $\mathrm{x}_{1}$, power costs of unit wastewater $\left(\mathrm{kWh} \cdot \mathrm{m}^{-3}\right) ; \mathrm{x}_{2}$, sludge disposal costs $\left(\mathrm{Y} \cdot \mathrm{t}^{-1}\right)$.

Relationship between sludge retention time and unit power consumption: Sludge retention time (SRT) is one of the most fundamental and important parameter in the design and operation of biological nutrient removal activated sludge systems and which refers to the average sludge retention time of activated sludge in the wastewater treatment plant. Generally, the better quality of the effluent and waste sludge required the longer sludge retention time, the larger biological reactor and the more wastewater characteristics. However, the effluent quality will descend sharply if the sludge retention time is less than a certain value. Hence, the sludge retention time is often suggested longer than designed parameter, more than 10 days often can be adopted in biological nitrification system. With the variety of sludge retention time, dissolved COD in effluent changed a little, which results in the effluent COD and BOD tending to be low as the same time keeping stable.

The shorter sludge retention time corresponds to less hydraulic retention time and the lower the oxygen demand, when the quantity and quality of influent wastewater is in a relatively stable state. On the contrary, the longer sludge retention time need the longer hydraulic retention time and the higher oxygen demand.

Fig. 2 shows the relationship between sludge retention time and power costs per ton based on the data obtained from the wastewater treatment plant as stated above.

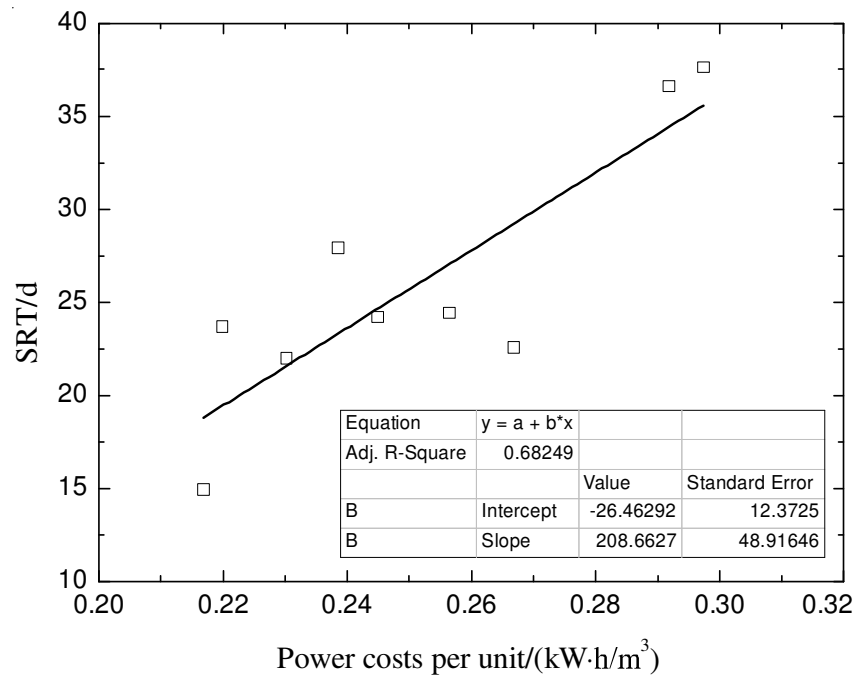

Fig. 2. Relationship between sludge retention time and power costs

Power costs per unit is approximately proportional to sludge retention time within a certain range, the function for this is given by eqn. (2):

Sludge retention time $($ SRT $)=208.66 \times \mathrm{x}_{1}-26.46$

Relationship between wastewater treatment costs and wastewater flow rate: The daily ultimate sludge disposal production is excess and the function for sludge production produced by unit wastewater is given by eqn. (3):

$$
\mathrm{b}=\mathrm{VX} / \mathrm{SRT} / \mathrm{Qr}
$$

where $\mathrm{V}$ is aeration tank volume $\left(\mathrm{m}^{3}\right) ; \mathrm{X}$ is mixed liquor suspended solid $\left(\mathrm{mg} \cdot \mathrm{L}^{-1}\right)$; Q is wastewater capacity $\left(\mathrm{m}^{3} \cdot \mathrm{d}^{-1}\right) ; \mathrm{r}$ is sludge total solid ratio (\%).

Based on the above analysis, the function between the costs of wastewater treatment per unit and power costs per unit and costs of sludge disposal produced by unit wastewater is given by eqn. (4):

$$
\begin{aligned}
& \mathrm{T}=\mathrm{ax}_{1}+\mathrm{bx}_{2}=\mathrm{ax}_{1}+(\mathrm{VX} / \mathrm{SRT} / \mathrm{Qr}) \mathrm{x}_{2} \\
& =\mathrm{ax}_{1}+\left(\mathrm{VXx}_{2} /\left(208.66 * \mathrm{x}_{1}-26.46\right) / \mathrm{Qr}\right.
\end{aligned}
$$

where $\mathrm{a}, \mathrm{b}, \mathrm{x}_{1}, \mathrm{x}_{2}$ have the same definition as stated in Experimental section. Substituting actual data back into the eqn. (4) will obtain eqn. (5): 


$$
\begin{aligned}
& \mathrm{T}=0.62 *(0.8533 / \mathrm{x}+0.1457)+5.22 / \\
& (175.87 *(0.8533 / \mathrm{x})+0.1457)-18.837 / \mathrm{x}
\end{aligned}
$$

According to the eqn. (5), simulated value of wastewater per unit costs will be easily attained.

Statistical test: In order to use homogeneity of variance test (F test) to confirm the accuracy of the eqn. (5) based on the data from Table-1, we check the critical value $F_{a}=12.25$ (degrees of freedom are respectively 1 and 7 gained form Table-1, degree of confidence is a $=0.01$ ) and $\mathrm{F}$ test value of the eqn. (5) is 24.8. Clearly, $\mathrm{F}>\mathrm{F}_{\mathrm{a}}$, thus the model can be

\begin{tabular}{|c|c|c|c|c|c|}
\hline \multicolumn{6}{|c|}{$\begin{array}{c}\text { TABLE-1 } \\
\text { POWER COSTS PER UNIT (PCPU) FOR CONTINUOUSLY } \\
\text { OPERATING } 9 \text { MONTHS }\end{array}$} \\
\hline Month & 4 & 5 & 6 & 7 & 8 \\
\hline PCPU $\left(\mathrm{kWh} \cdot \mathrm{m}^{-3}\right)$ & 0.1996 & 0.1961 & 0.1814 & 0.1895 & 0.1625 \\
\hline Month & 9 & 10 & 11 & 12 & \\
\hline PCPU $\left(\mathrm{kWh} \cdot \mathrm{m}^{-3}\right)$ & 0.1778 & 0.1817 & 0.1699 & 0.1714 & \\
\hline
\end{tabular}
verified availably with $\mathrm{F}$ test.

Simulated values which represent the relationship between the costs of wastewater treatment per unit and flow rate can be received though eqn. (5), which are shows in Fig. 3:



Fig. 3. Relationship between costs of wastewater treatment per unit and flow rate

Fig. 3 indicates that within a flow range of design, the direct treatment costs will reduce as the increasing of unit wastewater treatment quality. The main reason is that part of equipment energy consumption is insignificant with quality of wastewater disposal changes, so the running boundary costs will reduce as the increasing of wastewater treatment in the wastewater treatment plant.

Total costs analyses: In accordance with eqn. (4), we can calculate electricity price parameters according to actual situation of peak and valley price. Then, the price is 0.91 $\mathrm{Yok}^{-1} \cdot \mathrm{W}^{-1} \cdot \mathrm{h}^{-1}$ (peak value) and $0.33 \mathrm{Y} \cdot \mathrm{k}^{-1} \cdot \mathrm{W}^{-1} \cdot \mathrm{h}^{-1}$ (valley value). Figs. (4) and (5) show the relationship between total costs of wastewater treatment and power costs and sludge disposal costs.

Fig. 4 shows the situation of electricity value at the peak, the electricity price is higher than power level value, lower sludge retention time should be applied which can reduce the amount of the aeration and energy consumption and make total running costs relatively lower. In contrast, extended aeration will greatly improve the amount of the aeration, consequently make the total running costs relatively higher than present method. So in electricity peak stage, on condition that effluentquality standard can be guaranteed, lower sludge retention time and lower aeration quantity can be used in order to save costs. Sludge stability may not be considered in this stage.

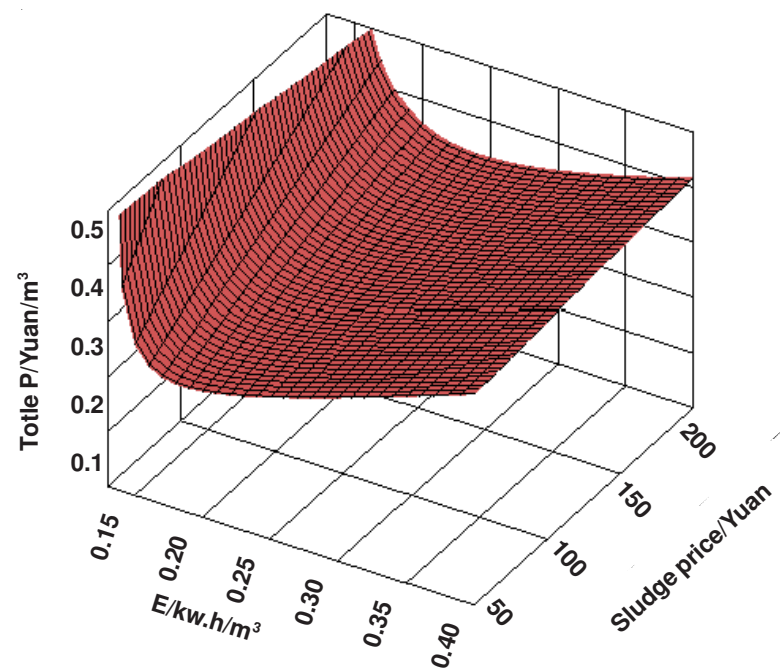

Fig. 4. Relationship between total costs of wastewater treatment and power costs and sludge disposal costs (peak)

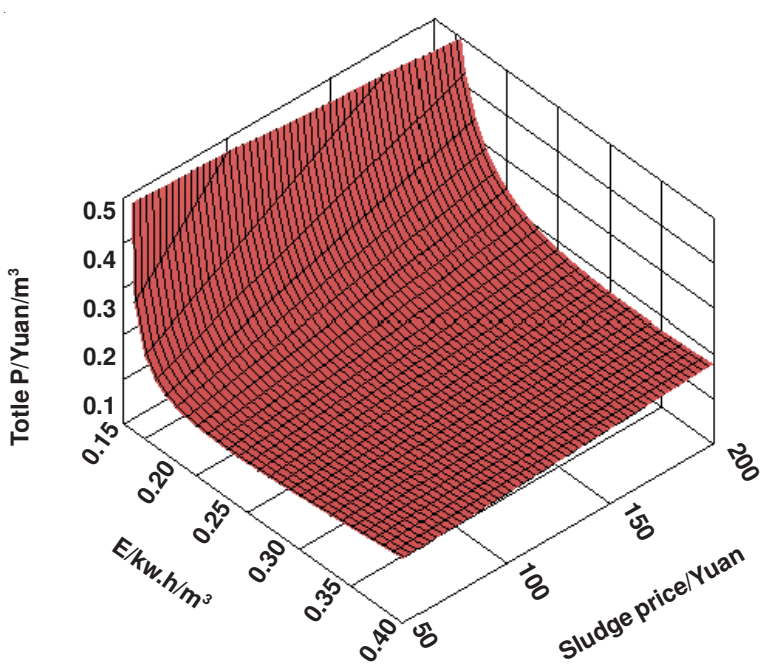

Fig. 5. Relationship between total costs of wastewater treatment and power costs and sludge disposal costs (valley)

Fig. 5 shows the situation of electricity value at the valley, the electricity price is much lower than power level value, only $0.2 \sim 0.4 \mathrm{kWh} \cdot \mathrm{m}^{-3}$. At this point, energy consumption will mount up as increasing of power costs per unit. And with the increasing of power costs per unit, sludge quality will reduce, thus the total costs only increase by a slight. So in electricity valley stage, extended aeration method can be applied in order to make the sludge aerobic stable in anaerobic tank and sludge production lower in wastewater treatment system which can reduce the sludge disposal costs. In this sense, the overall running cost isn't change greatly due to lower electricity price and reduction of sludge production. 
Moreover, as it is illustrated in the model, when sludge disposal costs are much higher, extended aeration and long sludge retention time should be considered, which can lead to sludge aerobic stable in anaerobic tank, i.e., it can reduce the sludge production. Instead, low aeration and short sludge retention time can be applied in order to make the total costs as low as possible when sludge disposal price is cheap.

It is obvious from the preceding discussion that the total costs of wastewater treatment only take power costs and sludge disposal costs into account, without considering sludge disposal methods. As sludge disposal methods coming into its own, wastewater and sludge co-treatment will have a strangely high priority and influence in future research field.

\section{Conclusion}

Under the ever-increasing global energy crisis, the need for improving operating ability of wastewater treatment plants in terms of cost and performance has never been stronger than before. This paper established the mathematical model about the correlation between sewage treatment cost and unit power consumption and sludge disposal costs and passed the statistics test. The results demonstrated that: (1) the key factors which effected plant total costs were power consumption and sludge disposal costs; (2) Unit power consumption was approximately proportional to sludge retention time within a certain range; (3) Within a flow range of design, the marginal operating costs reduced as the increasing of sewage quality in the sewage treatment plant; (4) The mathematical model of the relationship between sewage treatment costs and power consumption and sludge disposal costs was established. It indicated that we should adopt a large amount of aeration, high sludge retention time, thus the sludge will stabilize in the aeration tank when the electricity was in valley. On the contrary, lower aeration rate and lower sludge retention time will be adopted to achieve the lowest total costs of processing during the electricity in peak.

\section{REFERENCES}

1. J.-H. Ryu, T.-J. Kim, T.-Y. Lee and I.-B. Lee, J. Taiwan Inst. Chem. Eng., 41, 506 (2010).

2. W.H. Shen, X.Q. Chen, M.N. Pons and J.P. Corriou, Chem. Eng. J., 155, 161 (2009).

3. T. Hashimoto, K. Onda, Y. Nakamura, K. Tada, A. Miya and T. Murakami, Water Res., 41, 2117 (2007).

4. S. Xia and J. Liu, Process Biochem., 39, 1111 (2004).

5. Y. Zhang, Z. Li, Y.H. Song and H. Wang, Chin. J. Environ. Eng., 6, 945 (2012).

6. U. Jeppsson and M. Pons, Contr. Eng. Pract., 12, 299 (2004).

7. G.L. Liu, Y.X. Chong and Q.J. Fan, Chin. J. Environ. Sci., 27, 2323 (2006) (in Chinese).

8. G. Olsson, M. Nielsen, Z. Yuan, A. Lynggaard-Jensen and J.P. Steyer, Instrumentation, Control and Automation in Wastewater Systems, London: IWA Publishing (2005).

9. R.J. Wai and M.C. Lee, IEEE Transact. Indust. Elect., 51, 201 (2004).

10. S.J. Kenway, P.A. Lant, A. Priestley and P. Daniels, Water Sci. Technol., 63, 1983 (2011).

11. J. Chang, A.-M. Yang, Y.P. Gan, C.-L. Meng, Y.-Z. Peng, S.J. Zhang and Q.F. Ying, China Water and Wastewater, 27(4), 33 (2011) (in Chinese).

12. H.X. Hou, H.C. Shi, Y.Z. Wang, G. Wang and R.H. Xie, Water and Wastewater Eng., 35, 34 (2009) (in Chinese).

13. X. Gao, T.R. Long and J.S. Guo, J. Chongqing Univ. (Nat. Sci. Ed.). 25, 143 (2002) (in Chinese).

14. S.S. Yang, China Water and Wastewater, 6, 15 (1984) (in Chinese). 